\title{
Atomistic Simulation of Ice Nucleation on Silver lodide (0001) Surfaces with Defects
}

\section{Vahabpour Roudsari, Golnaz}

2020-01-09

Vahabpour Roudsari , G, Reischl , B , Pakarinen, O H \& Vehkamäki , H 2020 , ' Atomistic Simulation of Ice Nucleation on Silver lodide (0001) Surfaces with Defects ' , Journal of Physical Chemistry C , vol. 124 , no. 1, pp. 436-445 . https://doi.org/10.1021/acs.jpcc.9b08502

http://hdl.handle.net/10138/322704

https://doi.org/10.1021/acs.jpcc.9b08502

unspecified

acceptedVersion

Downloaded from Helda, University of Helsinki institutional repository.

This is an electronic reprint of the original article.

This reprint may differ from the original in pagination and typographic detail.

Please cite the original version. 


\section{Atomistic Simulation of Ice Nucleation on Silver lodide (0001) Surfaces with Defects}

Golnaz Roudsari, Bernhard Reischl, Olli H. Pakarinen,* and Hanna Vehkamäki

Institute for Atmospheric and Earth System Research / Physics, University of Helsinki, PO Box 64, FI-00014, Finland

E-mail: olli.pakarinen@helsinki.fi 


\begin{abstract}
Small particles of silver iodide (AgI) are known to have excellent ice nucleating capabilities and have been used in rain seeding applications. It is widely believed that the silver terminated (0001) surface of $\beta$-AgI acts as a template for the basal plane of hexagonal ice. However, the (0001) surface of ionic crystals with the wurtzite structure is polar and will therefore exhibit reconstructions and defects. Here, we use atomistic molecular dynamics simulations to study how the presence of defects on AgI (0001) affects the rates and mechanism of heterogeneous ice nucleation at moderate supercooling at $-10^{\circ} \mathrm{C}$. We consider AgI (0001) surfaces exhibiting vacancies, step edges, terraces, and pits, and compare them to simulations of the corresponding ideal surface. We find that while point defects have no significant effect on ice nucleation rates, step edges, terraces and pits reduce both the nucleation and growth rates by up to an order of magnitude. The reduction of the ice nucleation rate correlates well with the fraction of the surface area around the defects where perturbations of the hydration layer hinder the formation of a critical ice nucleus.
\end{abstract}

\title{
Introduction
}

The presence of a seed particle can drastically reduce the supercooling needed for freezing water compared to homogeneous nucleation. This heterogeneous ice nucleation is ubiquitous in nature and plays an important role in cloud formation. For example, mineral dust and organic material can serve as ice nucleation particles (INPs). The mechanism of heterogeneous ice nucleation depends on a balance between surface morphology and hydrophobicity. ${ }^{1}$ On most mineral dust particles, there are only a few active sites for ice nucleation, typically around defects or pits. ${ }^{2-4}$

Silver iodide (AgI) particles are very effective INPs, used for rain seeding, ${ }^{5}$ and they have been studied both experimentally (see the review by Marcolli et al. ${ }^{6}$ ) and computationally, focusing on ice nucleation on flat AgI surfaces, ${ }^{7,8} \mathrm{AgI}$ disks and plates, ${ }^{9}$ the effect of surface 
charge distribution, ${ }^{10}$ the effect of defect surface fraction, ${ }^{11}$ as well as water adsorption in slit-like AgI pores. ${ }^{12}$ In ambient conditions, both AgI crystals with the wurtzite structure $(\beta$-AgI $)$ and the zincblende structure $(\gamma-\mathrm{AgI})$ are stable. Previous simulations have found that ice could nucleate at the silver terminated (001) and (111) surfaces of $\gamma$-AgI, and the silver-terminated (0001) surface of $\beta$-AgI. ${ }^{8}$ The ice nucleating ability of AgI has been attributed to the (0001) surface of $\beta$-AgI acting as a very good template for the basal plane of hexagonal ice (ice $I_{h}$ ) or the equivalent (111) plane of cubic ice (ice $\left.I_{c}\right),{ }^{9}$ with a lattice mismatch of approximately $2 \%$ as illustrated in Figure 1. However, $\mathrm{AgI}$ (0001) is a type 3 polar surface, ${ }^{13}$ and therefore intrinsically unstable. While a recent study showed that the interface with an aqueous solution can stabilise the (0001) surface, ${ }^{14}$ reconstructions and defects are still expected to be abundant, as can be seen from electron microscope images of micrometer-sized AgI plate crystals exposing (0001) facets. ${ }^{15}$ It is therefore important to investigate how the presence of such defects on AgI (0001) surfaces affects ice nucleation and growth rates. For many other systems, ice nucleates on time scales that are hard to reach in an unbiased atomistic molecular dynamics simulation, making it necessary to utilize a coarse grained approach using the monatomic $\mathrm{mW}$ model of water, and/or more elaborate simulation techniques, such as seeded nucleation simulations, ${ }^{16}$ or advanced sampling techniques such as forward-flux sampling ${ }^{17}$ or transition path sampling. ${ }^{18}$ In contrast, ice nucleation on AgI typically starts on a timescale of nanoseconds, ${ }^{8,9}$ easily accessible in an unbiased molecular dynamics simulation, even for large system sizes.

In this work, we use molecular dynamics (MD) simulations to study the effect of defects, such as vacancies, step edges, terraces and pits, on ice nucleation on AgI (0001) surfaces. 
(a) Ice Ih, basal plane

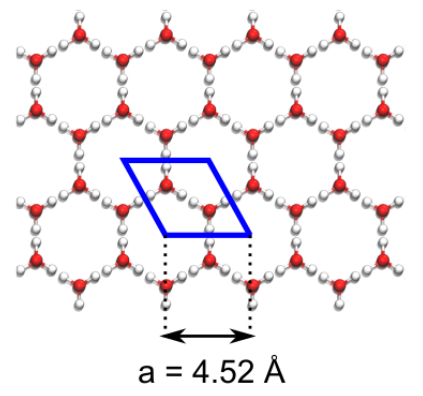

(b) B-Agl (0001) surface

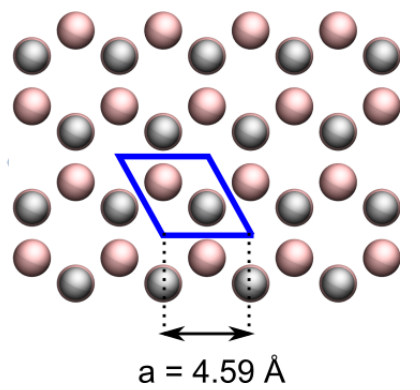

Figure 1: The basal plane of ice $\mathrm{I}_{\mathrm{h}}$ (a) and the (0001) surface of $\beta$-AgI (b) seen along the c-axis of the crystals. The lattice mismatch is less than $2 \%$. O , H, Ag, and I are colored in red, white, silver, and pink, respectively. The hexagonal unit cells are indicated by blue lines.

\section{Methods}

\section{Force field and Molecular Dynamics details}

Several force fields have been fitted to reproduce bulk crystal structures and the phase diagram of silver iodide (AgI). ${ }^{19,20}$ However, the perfect (0001)-terminated AgI slab has an intrinsic dipole moment and none of these force fields yields a stable surface. In previous ice nucleation simulations, atoms in the AgI surface have therefore either been fixed to bulk positions, or constrained by a spring potential. In the present work, all AgI surfaces considered are bulk-terminated and atoms are completely fixed at the positions of an ideal wurtzite crystal with lattice constants $a=4.58 \AA$ and $c=7.50 \AA$. The silver and iodide ions only interact with the water molecules, described by the TIP4P/Ice model. It reproduces the properties of bulk liquid water very well, and yields the correct melting point and coexistence curve between water and ice I at low pressures. ${ }^{21}$ The interactions between $\mathrm{Ag}^{+}$and $\mathrm{I}^{-}$ions and TIP4P/Ice water molecules are described by the potential of Hale and Kiefer. ${ }^{22}$ The ions carry a point charge and a Lennard-Jones interaction with the oxygen atom of water, OW. The Lennard-Jones parameters are $\epsilon_{\mathrm{Ag}-\mathrm{OW}}=2.289 \mathrm{~kJ} / \mathrm{mol}, \sigma_{\mathrm{Ag}-\mathrm{OW}}=3.17 \AA$, $\epsilon_{\mathrm{I}-\mathrm{OW}}=2.602 \mathrm{~kJ} / \mathrm{mol}, \sigma_{\mathrm{I}-\mathrm{OW}}=3.34 \AA$ for the Ag-OW and I-OW interactions, respectively. Zielke et al. have previously shown that changing the value of the partial charges between 
$\pm 0.2 \mathrm{e}$ and $\pm 0.6 \mathrm{e}$ does not have any considerable effect on ice nucleation results on $\gamma$-AgI, ${ }^{8}$ hence we also use the original value of $\pm 0.6 \mathrm{e}$ from Hale and Kiefer in the present simulations. We do not consider any polarization effects in our electrostatic interactions, since the TIP4P/Ice water model also has a rigid point charge geometry. ${ }^{8}$ The AgI-water interactions were originally fitted in conjunction with a water model based on the ST-2 potential by Stillinger and Rahman. ${ }^{23}$ Since we are using a different water potential, we chose to reduce the cut-off distance in the AgI-water interactions to match the cut-off used in the TIP4P/Ice potential. All molecular dynamics (MD) simulations were performed using the GROMACS software version $5 .^{24,25}$ The equations of motion were integrated using the leapfrog algorithm with a time step of $2 \mathrm{fs}$. Lennard-Jones and real-space electrostatic interactions were cut off at $8.5 \AA$. The particle mesh Ewald (PME) method ${ }^{26}$ was used to calculate long-range electrostatic interactions. The SETTLE algorithm ${ }^{27}$ was used to constrain the O-H bond length and $\mathrm{H}-\mathrm{O}-\mathrm{H}$ angle of the water molecules. In NVT production runs, the temperature was controlled with a Nosé-Hoover thermostat ${ }^{28}$ with a relaxation time of 0.4 ps. For NPT equilibration runs, a Berendsen thermostat and barostat, with time constants of 0.4 ps and 5 ps respectively, were used.

\section{Simulation setup and treatment of surface dipole}

As previously mentioned, the (0001) surface of AgI has an intrinsic dipole moment which makes this surface challenging to simulate. We have therefore carried out preliminary checks on how different simulation setups, illustrated in Figure 2a-d, affect the behaviour of water at the interface, to avoid artefacts in the ice nucleation studies. For simplicity, we only considered the perfect (0001) surface of $\beta$-AgI at a temperature $T=273 \mathrm{~K}$. The most common setup for simulating a solid-liquid interface is to have a solid slab in contact with a volume of water in a simulation box with 3D periodic boundaries (see Fig. 2a). Throughout this work, the ice surface is always considered to lie in the $\mathrm{x}, \mathrm{y}$ plane and $\mathrm{z}$ is the direction perpendicular to the surface. In order to equilibrate the system, i.e. form hydration layers at 
the interface and reach bulk water density in the central region of the liquid between the two interfaces, one can carry out an NPT simulation were the box vectors along $\mathrm{x}$ and $\mathrm{y}$ are fixed to the values determined by the crystal's lattice constant, but the box vector along z can fluctuate. Allowing for a change of water density is essential during nucleation simulations as well, since the density of water and ice in the TIP4P/Ice water model, are $0.985 \mathrm{~g} / \mathrm{ml}$ and $0.906 \mathrm{~g} / \mathrm{ml}$, respectively. ${ }^{29}$ In the case of AgI (0001), this traditional setup does not work, as the dipole field leads to attractive forces between periodic images of the slab, "squeezing" the water and significantly increasing its density above the equilibrium value (see Fig. 2e). To avoid this artefact, the crystal slab can instead be in contact with sufficiently thick films of water on either side (see Fig. 2b). An appropriately chosen vacuum gap between the two films' interfaces on the opposite side, together with a simulation in NVT conditions, can ensure that the density of the liquid reaches the proper value in the 'bulk' region between the interfaces with the solid or the vacuum (see Fig. 2e). However, the dipole field still causes a spurious alignment of water molecules far away from the surface (see Fig. 2f). A 'mirror

image' geometry can be used to effectively cancel the dipole field: two copies of the AgI (0001) slab, mirrored along the x,y plane, are placed on opposite ends of the simulation box, each in contact with a water film with an open surface, and separated by a vacuum gap (see Fig. 2c). This setup ensures the correct bulk water density and removes the spurious water orientation (see Fig. 2e and f). Finally, to reduce the number of atoms in the simulation, we have also considered a mirror image setup of the crystal slabs, but with the water on the upper slab removed (see Fig. 2d). This setup gives results identical to the previous setup (see Fig. 2e and f) and was therefore chosen for all the nucleation simulations performed in this work.

\section{Silver iodide surfaces with defects}

To study the effect of defects on ice nucleation at the silver iodide surface, we consider charge neutral AgI (0001) surfaces with single or double vacancies, a step edge along [210] and [100], 
(a)

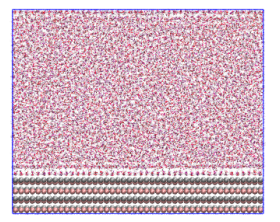

(b)

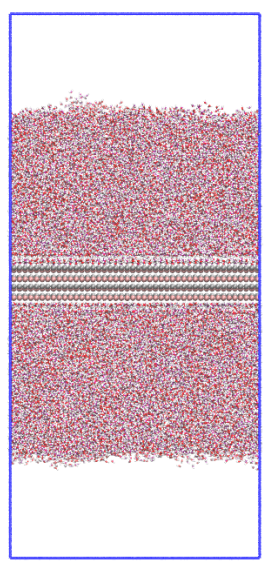

(c)

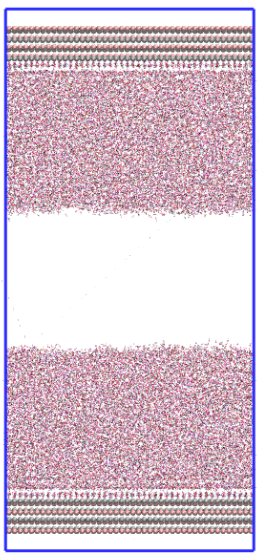

(d)

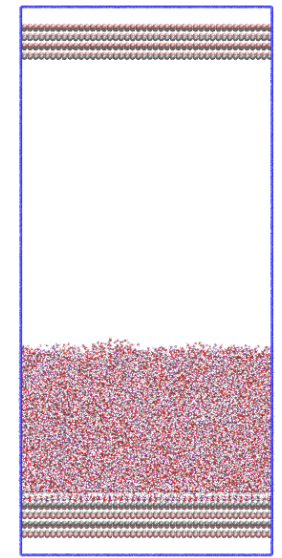

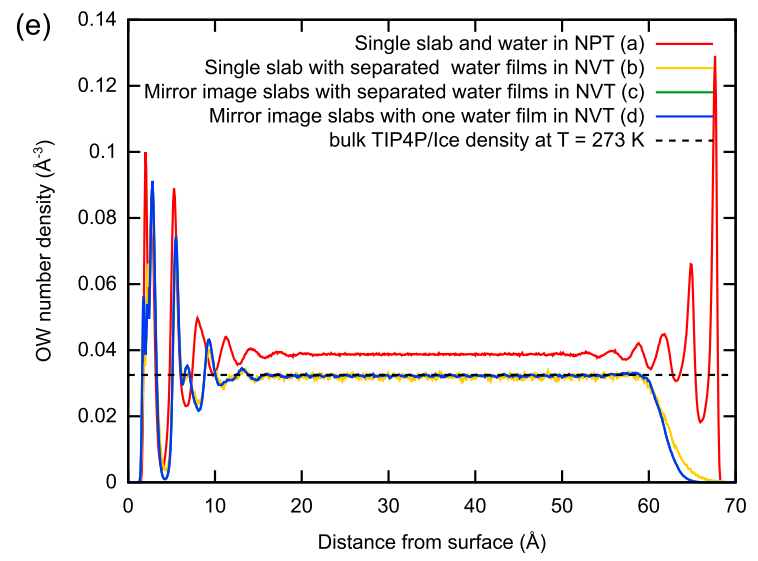

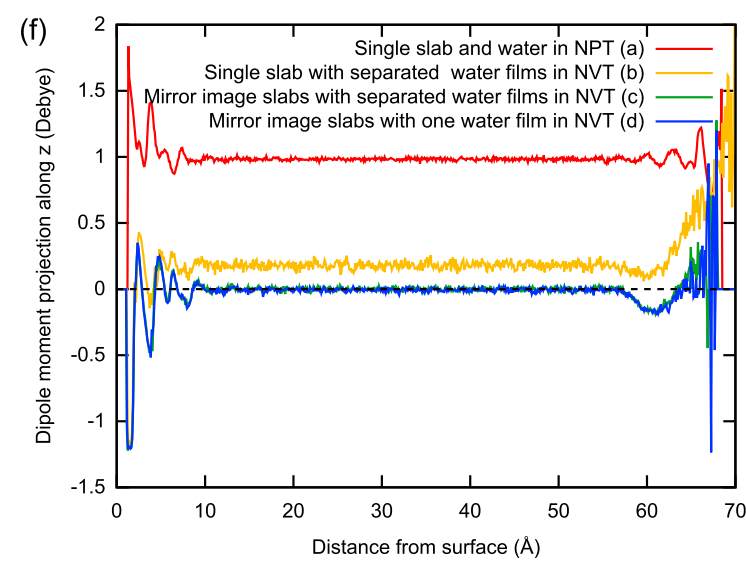

Figure 2: Different simulation setups for an AgI (0001)-water interface (top): (a) slab in contact with bulk water in 3D PBC, (b) slab in contact with two films of water with open surfaces, (c) mirror image geometry with two films of water and (d) mirror image geometry with only one film of water. (e) water density profile along $\mathrm{z}$ and (f) profile of the projection of the water dipole moment along z observed for the different simulation setups (bottom).

terraces, and pits, and compare them to simulations of the corresponding ideal surface. The six different non-ideal geometries are illustrated in Fig. 3. In the double vacancy system, an ion pair was removed from the top of the surface, whereas in the single vacancy system, an $\mathrm{Ag}^{+}$ion was removed from the top surface, and an $\mathrm{I}^{-}$ion was removed from the bottom to keep the system charge neutral. In all systems, [100] and [210] crystallographic directions correspond to $\mathrm{x}$ and $\mathrm{y}$ directions of the simulation cell, respectively. The mirror image geometry, with water only present on top of the lower AgI slab, was used (see Fig. 2d). The open water surface allows for a change of water density during ice nucleation, even though the simulation box volume is constant. To minimize finite size effects, such as the interaction 
of the ice nucleus with itself through the periodic boundaries along $\mathrm{x}$ and $\mathrm{y}$, the lateral dimensions of the simulation box are chosen to be quite large $\left(100.76 \times 103.13 \AA^{2}\right)$. Also, the film of water on the lower AgI slabs has a thickness of $\sim 60 \AA$, to have a considerable number of bulk-like water molecules and to be able to observe the growth of many layers of ice after the onset of nucleation. The total height of the simulation boxes was $220 \AA$.

(a) Single vacancy

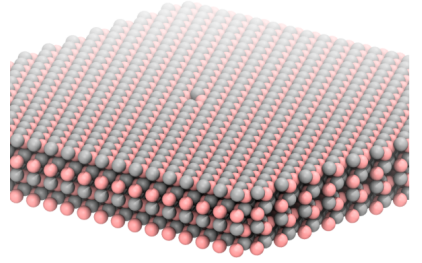

(c) Step edge [100]

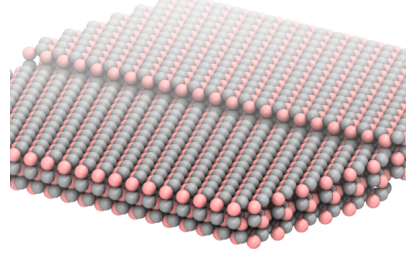

(e) Terrace

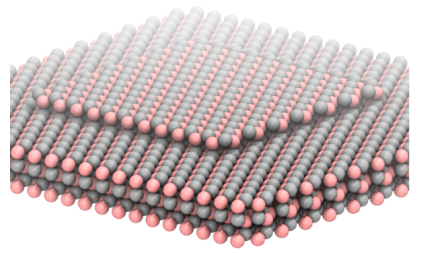

(b) Double vacancy

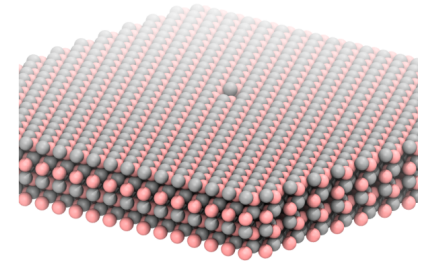

(d) Step edge [210]

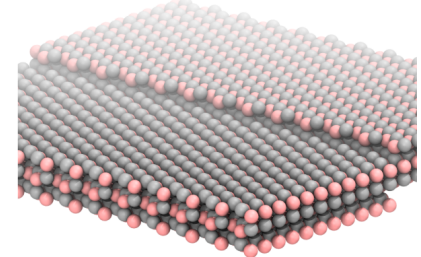

(f) Pit

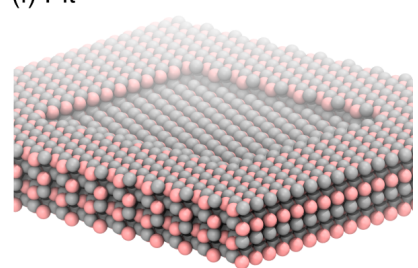

Figure 3: Atomistic models of six different AgI (0001) surfaces with defects. $\mathrm{Ag}^{+}$and $\mathrm{I}^{-}$ ions are colored in silver and pink, respectively.

\section{Results and discussion}

To study ice nucleation and growth rates on the perfect surface and surfaces with defects, we analyse snapshots of the MD trajectories taken at every nanosecond using the CHILL+ algorithm ${ }^{30}$ to classify each $\mathrm{H}_{2} \mathrm{O}$ molecule as cubic ice, hexagonal ice, interfacial ice, or liquid, based on its hydrogen bond arrangement. The final frames of each trajectory are shown in Figure S1 in the Supporting Information. By identifying the appearance of critical nuclei 
and determining the volume of ice formed during the simulations, we are able to determine the ice nucleation and growth rates for each system.

\section{Nucleation rates}

To quantify the effectiveness of the different systems for ice nucleation, we calculate nucleation rates at $T=263 \mathrm{~K}$ following the procedure presented by Cox et al. ${ }^{31}$ By monitoring the size of the largest ice cluster in the system using the CHILL+ algorithm, the induction time is found, which is defined as the time when the ice cluster starts growing monotonously. For each system, 10 individual NVT simulations are started with different initial configurations and velocities of the water molecules. The induction times observed are used to calculate the probability $P_{\text {liq }}(t)$ of an ensemble of systems to be still in the liquid state after time $t$. As the temperature is $10^{\circ} \mathrm{C}$ below the melting point, we expect the probability to decay exponentially. We fit the data using equation

$$
P_{\text {liq }}(t)=\exp \left[-(R t)^{\gamma}\right]
$$

where $\mathrm{R}$ shows the decay rate, equal to the nucleation rate, and the exponent $\gamma$ is a second fitting parameter, as illustrated in Figure 4.

The nucleation rates obtained for the different surfaces are shown in Table 1. The standard errors were obtained from the least squares error of the fit using Eq. 1. The surface with a double vacancy and the perfect surface exhibit the highest nucleation rates with values of $3.89 \times 10^{23} \mathrm{~m}^{-2} \mathrm{~s}^{-1}$ and $3.86 \times 10^{23} \mathrm{~m}^{-2} \mathrm{~s}^{-1}$, respectively. Surfaces with a single Ag vacancy show almost the same nucleation rate $\left(3.75 \times 10^{23} \mathrm{~m}^{-2} \mathrm{~s}^{-1}\right)$. The nucleation rates on surfaces with point defects are very similar to the rate on the perfect surface. The two step edges considered affect the nucleation rate with different severity, leading to rates of $1.06 \times 10^{23} \mathrm{~m}^{-2} \mathrm{~s}^{-1}$ and $0.47 \times 10^{23} \mathrm{~m}^{-2} \mathrm{~s}^{-1}$ for the step edge along [100] and [210] directions, respectively. Finally, nucleation rates of $1.41 \times 10^{23} \mathrm{~m}^{-2} \mathrm{~s}^{-1}$ and $1.09 \times 10^{23} \mathrm{~m}^{-2} \mathrm{~s}^{-1}$ were 


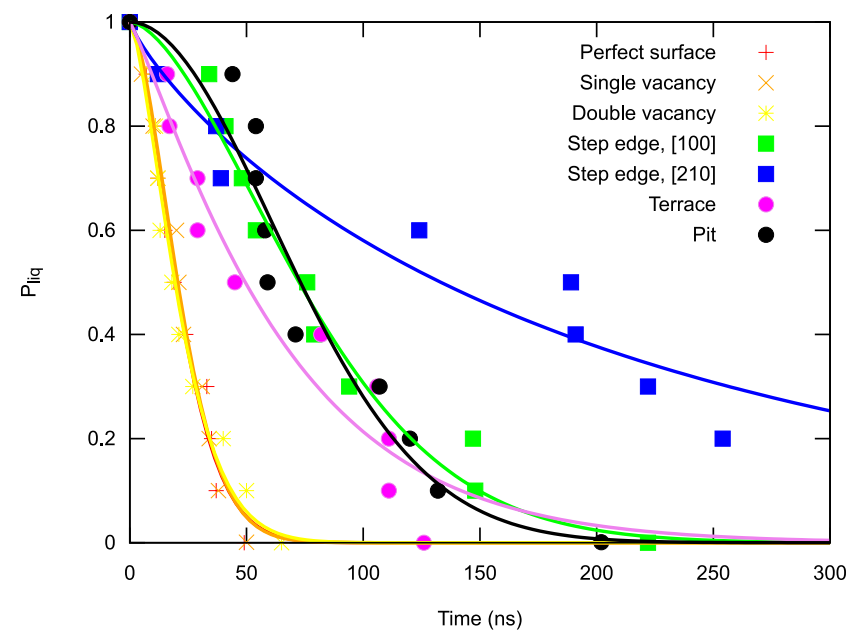

Figure 4: Obtaining ice nucleation rates on different AgI (0001) surfaces from fitting the induction times for ice nucleation in 10 independent molecular dynamics simulations at $T=263 \mathrm{~K}$.

observed for the surface with a terrace, or a pit, respectively.

Table 1: Ice nucleation rates and uncertainties on different AgI (0001) surfaces with defects at $T=263 \mathrm{~K}$.

\begin{tabular}{cc}
\hline System & Nucleation rate $\left(\times 10^{23} \mathrm{~m}^{-2} \mathrm{~s}^{-1}\right)$ \\
\hline Perfect surface & $3.86 \pm 0.13$ \\
Single vacancy & $3.75 \pm 0.12$ \\
Double vacancy & $3.89 \pm 0.23$ \\
Step edge [100] & $1.06 \pm 0.04$ \\
Step edge [210] & $0.47 \pm 0.05$ \\
Terrace & $1.41 \pm 0.12$ \\
Pit & $1.09 \pm 0.06$ \\
\hline
\end{tabular}

These results are quite surprising, as for many surfaces ice nucleation rates are enhanced by the presence of defects. Here, however, the perfect surface is an excellent template for ice growth, due to the very small lattice mismatch between AgI (0001) and the basal plane, and all extended defects considered in this work reduce the nucleation rate. We also note that the nucleation rate on the perfect AgI (0001) surface reported here is roughly eight times smaller compared to a previous study by Glatz and Sarupria. ${ }^{10}$ However, the surface area in their simulation was less than $900 \AA^{2}$, compared to over $10000 \AA^{2}$ in the present study, which could indicate that their result was influenced by finite size effects. Finally, we also carried 
out simulations with similar systems at stronger undercooling at $T=258 \mathrm{~K}$ and $261 \mathrm{~K}$. At these temperatures, the induction times are even shorter and it becomes increasingly difficult to assess the influence of the defect on the nucleation rates.

\section{Nucleation mechanisms}

To better understand the ice nucleation mechanism at the silver iodide surface and, in particular, how the presence of defects affects the possibility to form a critical nucleus at the interface, we compare the hydration layer structure and dynamics at the perfect surface and around the step edge along the [210] direction.

On the perfect surface, the structure of the first hydration layer is very similar to the basal layer of ice $\left(\mathrm{I}_{\mathrm{h}}\right)$, as shown in Figure 5. Water oxygen atoms are situated at the corners of hexagons coordinating the protruding $\mathrm{Ag}^{+}$ion in the surface, i.e. directly on top of the sub-surface $\mathrm{I}^{-}$ion. At $273 \mathrm{~K}$, a well defined second hydration layer is also visible, before the changes in water density level off to the constant bulk water density.

To study the dynamics, residence times of water molecules in the first hydration layer were also calculated from MD trajectories using the method suggested by De La Pierre et al. ${ }^{32}$ We computed the molecules' survival function

$$
P(t)=\int_{t}^{\infty} \mathrm{E}\left(t^{\prime}\right) d t^{\prime}
$$

where $E(t) \mathrm{d} t$ represents the probability for a water molecule to remain coordinated to a surface Ag ion site for a time between $t$ and $t+\mathrm{d} t$. This was then fitted with the sum of two exponentially decaying functions,

$$
P(t)=a_{1} \exp \left(t / \tau_{1}\right)+a_{2} \exp \left(t / \tau_{2}\right)
$$

The small time constant $\tau_{1}$ corresponds to fluctuations in and out of the cut-off distance used to identify water molecules in the first hydration layer, and $\tau_{2}$ is the actual residence 

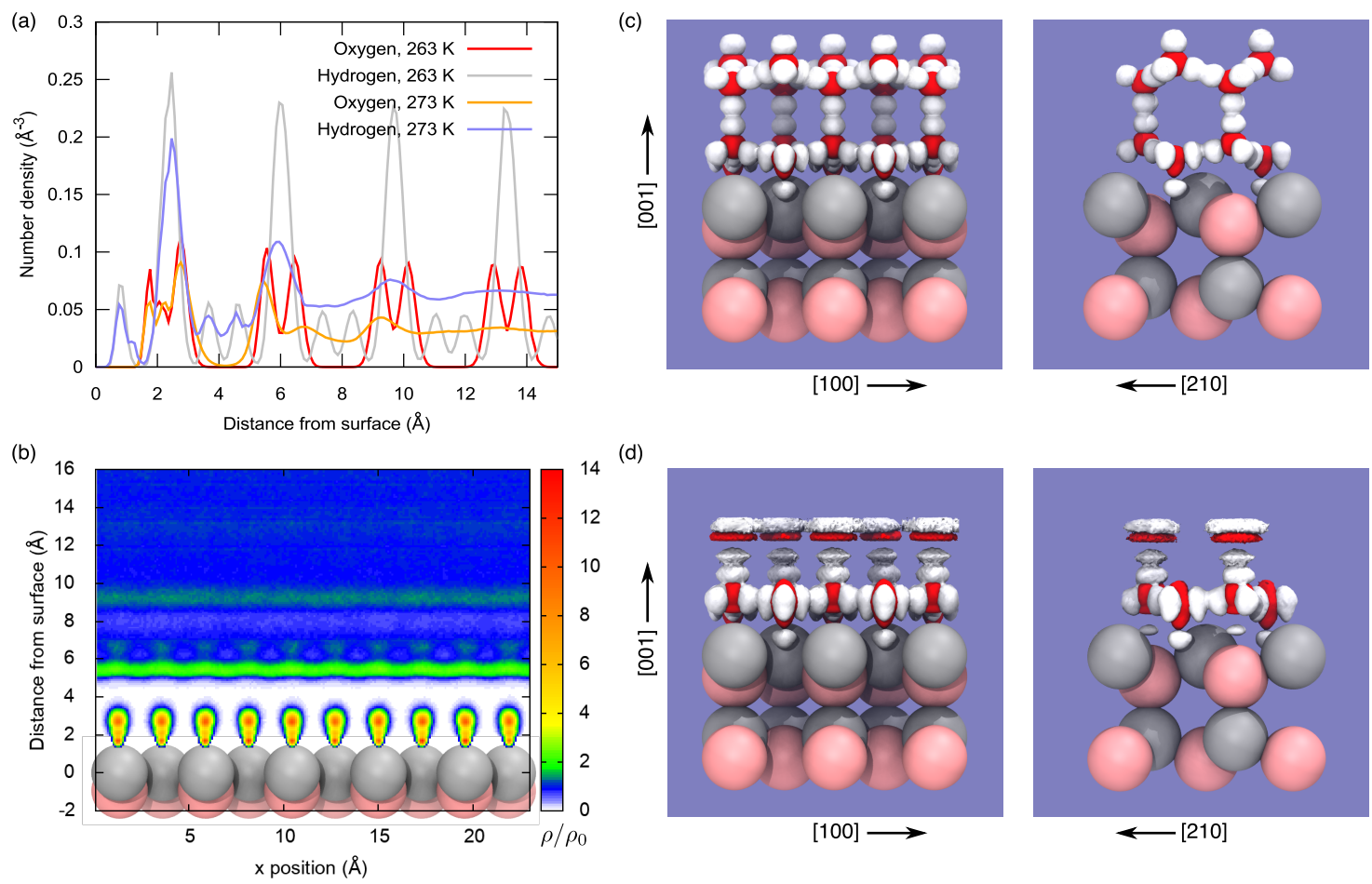

Figure 5: AgI (0001) hydration structure: (a) Number density of oxygen and hydrogen atoms as a function of the distance from the perfect AgI (0001) surface at $273 \mathrm{~K}$ and $263 \mathrm{~K}$. (b) Water oxygen atom density map projection in the xz plane over the AgI (0001) surface. The number density is expressed in units of the equilibrium water density at $T=273 \mathrm{~K}$, $\rho_{0} \approx 0.032 \AA^{-3}$, shown in blue. Surface $\mathrm{Ag}^{+}$and $\mathrm{I}^{-}$ions are colored in silver and pink, respectively. First and second hydration layer oxygen (red) and hydrogen (white) density isosurface plots over the perfect surface at $263 \mathrm{~K}$ (c) and $273 \mathrm{~K}(\mathrm{~d})$. The isosurfaces shown correspond to $\rho=25 \rho_{0}$. 
time of the water molecule before an exchange takes place.

While the equilibrium structure of the first hydration layer on the perfect surface is similar to the basal plane of ice $\mathrm{I}_{\mathrm{h}}$ and closely resembles the first layer of ice on the perfect surface at $263 \mathrm{~K}$ (see Fig. 5a), at $273 \mathrm{~K}$ the structure is still surprisingly dynamic, with water exchanging on a time scale of $\sim 2.0$ ns at $T=273 \mathrm{~K}$, as calculated from the survival probabilities in Eq. 2.

As can be seen in Figure 6a, the pattern of the first hydration layer is perturbed by the presence of the step edge, and the average time scale of water exchange is reduced to $\sim 1.8 \mathrm{~ns}$ on the surface containing the step edge. In order to quantify the spatial extent of the hydration layer perturbation around the step edge and link it to the observed nucleation rate, we have calculated the average deviation $d$ of first hydration layer water molecule oxygen from its ideal position, as a function of the position along $\mathrm{x}$, the direction perpendicular to the step edge (see Figure 6b),

$$
d=\sqrt{\left(x_{\mathrm{O}}-x_{\mathrm{I}}\right)^{2}+\left(y_{\mathrm{O}}-y_{\mathrm{I}}\right)^{2}}
$$

where $x_{\mathrm{O}}$ and $y_{\mathrm{O}}$ are the $\mathrm{x}$ and $\mathrm{y}$ positions of the oxygen atoms and $x_{\mathrm{I}}$ and $y_{\mathrm{I}}$ are the subsurface iodide ions' $\mathrm{x}$ and y positions. While on the perfect surface, the average disturbance of water patterning is only $\sim 0.3 \AA$, this value rises to a maximum $\sim 0.7 \AA$ over the step edge located at $x=50 \AA$. The spatial extent of the perturbation along $\mathrm{x}$ is $\pm 5.3 \AA$ on either side of the step edge. Values were averaged from three independent $10 \mathrm{~ns}$ long trajectories.

Combining the information about the spatial extent of hydration layer perturbation due to the presence of a defect with the average radius of the initial ice clusters at nucleation sites on the surfaces $\left(r_{c} \sim 10.3 \AA\right)$ allows us to build a very simple model to predict the reduction in nucleation rate compared to the perfect surface, based solely on the surface area excluded for the formation of a critical nucleus around a defect with a certain geometry. As can be seen in Figure 7, these predicted nucleation rates agree surprisingly well with the nucleation 


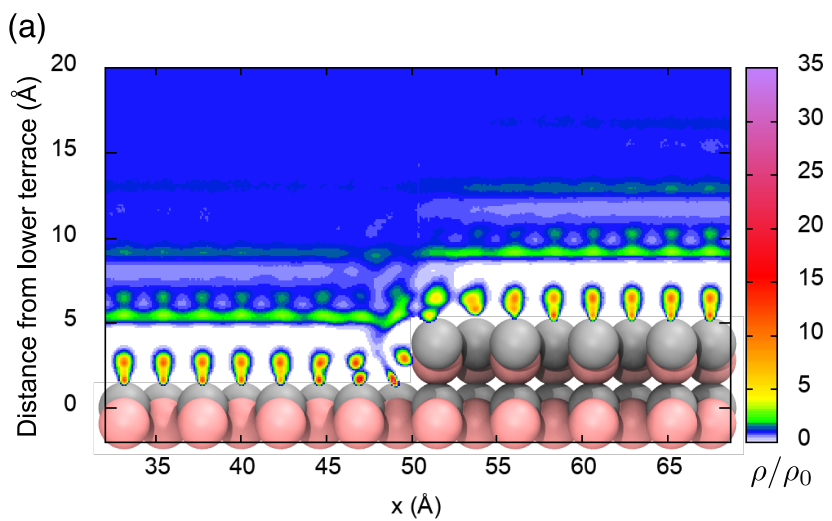

(b)

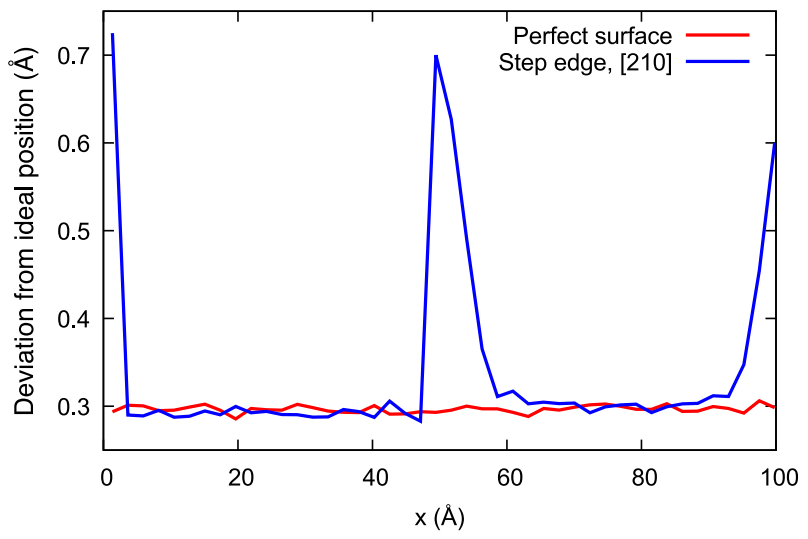

Figure 6: (a) Water oxygen atom density map projection in the xz plane over the AgI (0001) surface with a step edge along [210]. The number density is expressed in units of the equilibrium water density at $T=263 \mathrm{~K}, \rho_{0} \approx 0.032 \AA^{-3}$, shown in blue. Surface $\mathrm{Ag}^{+}$and $\mathrm{I}^{-}$ions are colored in silver and pink, respectively. (b) Disturbance of the water patterning in the first hydration layer with respect to the surface lattice, over the perfect surface (red line) and a surface with a step edge along [210] (blue line). 
rates obtained from molecular dynamics simulations. The result agrees qualitatively with a recent study, where ice nucleation on AgI (0001) was also shown to be suppressed by a large surface fraction of defects. ${ }^{11}$

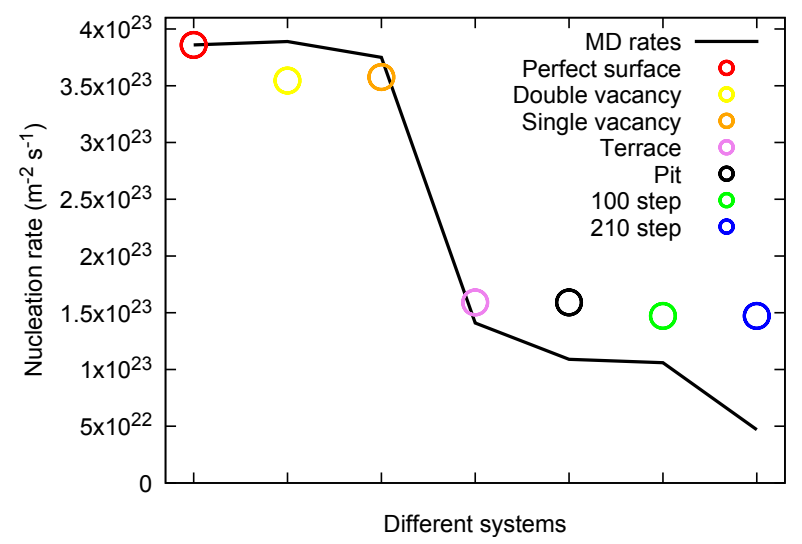

Figure 7: Ice nucleation rates obtained by scaling the nucleation rate on the perfect surface by the fraction of surface area exhibiting perfect water patterning (colored circles), compared to nucleation rates obtained from molecular dynamics simulations on the respective non-ideal surface (black line).

\section{Topological analysis of the interfacial hydrogen bond network}

In the recent study by Prerna et al., the surface fraction of defects on AgI (0001) has been shown to affect the hydrogen bond network topology, and the ratio of double-diamond cages (DDC) and hexagonal cages (HC). ${ }^{11}$ While this topological classification is very similar to the analysis using the CHILL+ algorithm in bulk ice, it is advantageous for the analysis of interfacial ice, as it can directly classify molecules which are not in a tetrahedral arrangement as belonging to $\mathrm{DDC}$ or $\mathrm{HC}$ cages. We have implemented the topological ring analysis by King ${ }^{33}$ and identified HC and DDC as previously described by Haji-Akbari and Debenedetti ${ }^{34}$ to study the hydrogen bond network topology in the two double layers adjacent to the AgI surfaces, in the last frame of each trajectory. The results of the analysis are summarised in Fig. S2 in the Supporting Information. We find that for the perfect surface and the surfaces with point defects, only either HC or DDC are present, with the former being more frequent (7/10 for perfect and single vacancy surfaces, $6 / 10$ for the surface with a 
double vacancy). For surfaces with terraces, ice nucleation starts on the flat surface around the terrace eight times out of ten. Here, 7/10 systems contain both HC and DDC, 3/10 systems contain only $\mathrm{HC}$ and ice nucleates 4 times as $\mathrm{HC}$ and 6 times as DDC. For surfaces with pits, ice nucleation always starts outside the defect. Each system shows both HC and DDC, in 9 cases only HC are present outside the defect and in one case only DDC, and the topology inside the pit is always opposite of the one on the flat surface outside. On the step edges along [100] ice always nucleates on the upper terrace, with HC and DDC occurring equally often. In one system only DDC are present, in two systems HC and DDC, in three systems HC and liquid water, and in four systems DDC and water. On the step edges along [210], we observed 7 nucleation events on the upper terrace, and one on the lower. In two cases the volumes of ice formed are still too small to determine a critical nucleus properly. However, all eight nucleation events occurred as HC. 5/10 systems contain only HC, one system contains both $\mathrm{HC}$ and DDC, and three systems $\mathrm{HC}$ and liquid water.

The topological analysis confirms that the presence of extended defects promotes the presence of both $\mathrm{DDC}$ and $\mathrm{HC}$ close to the surface, contrary to the perfect surface, or the two surfaces with point defects, where stacking faults between cubic and hexagonal ice do only appear at a later stage. The analysis also indicates that when comparing the step edges along [100] and [210], or the pit and terrace, which have the same spatial extents, the respective occurrences of $\mathrm{HC}$ and $\mathrm{DDC}$ are different. However, due to the number of different structures observes for each of these systems, and the relatively limited sample size, we cannot directly correlate the reduction in nucleation rate with e.g. the ratio of DDC and HC, as proposed by Prerna et al. ${ }^{11}$

\section{Ice growth rates}

We determined ice growth rates for the perfect surface and the surfaces with defects in terms of the volume of ice, or the thickness of the ice layer, grown per unit time. 


\section{Growth by volume}

To calculate the volume of ice, we determine the number of $\mathrm{H}_{2} \mathrm{O}$ molecules classified as hexagonal or cubic ice, $N_{\mathrm{C}+\mathrm{H}}$, in each frame of the trajectory using the CHILL+ algorithm and multiplying by the molecular volume of ice,

$$
V_{\text {ice }}=\frac{N_{\mathrm{C}+\mathrm{H}} N_{\mathrm{A}} M}{\rho_{\text {ice }}}
$$

where $N_{\mathrm{A}}$ is Avogadro's constant, $M$ is the molar mass of the water molecule and $\rho_{\text {ice }}$ is the density of ice. Examples of growth curves by volume can be found in Fig. 9 in the section on ice growth mechanism.

For each system, we determine the average growth rate from linear fits to the ice volume signal after the induction time, for the 10 individual simulations and normalize by the surface area. We can mostly observe a steady increase in the size of the nucleated ice clusters and eventual transition into layer-by-layer growth, once the clusters interact with themselves through periodic boundaries along $\mathrm{x}$ and $\mathrm{y}$. For each system, the 10 individual simulations with different initial configurations show roughly similar growth rates, but differences exist due to the stochastic appearance of stacking faults that in some cases can hinder the layerby-layer growth.

Average ice growth rates by volume and their standard error for all systems at $T=263 \mathrm{~K}$ are shown in Table 2. For unobstructed, layer-by-layer growth, such as on the perfect surface or the surface with a single or double vacancy, we can determine the highest growth rate of about $0.3 \AA \cdot \mathrm{ns}^{-1}$. This value is in good agreement with previous work ${ }^{7}$ carried out AgI (0001) surfaces at similar $10 \mathrm{~K}$ supercooling, and lower than the growth rate of $0.7 \AA \cdot \mathrm{ns}^{-1}$ obtained in a simulation of ice on ice growth. ${ }^{35}$ The slower growth on AgI has been attributed to the small lattice mismatch between the basal plane of ice $I_{h}$ and the (0001) surface of AgI. ${ }^{7,35}$ Experiments on a thin layer of ice show a growth rate of about $0.3 \AA \cdot \mathrm{ns}^{-1}$ at

$263 \mathrm{~K},{ }^{36,37}$ similar to the growth rate on the perfect AgI surface reported in this work. The 
systems with step edges along [100] and [210] directions both show similar growth rates of about $0.03 \AA \cdot \mathrm{ns}^{-1}$, although the [210] step shows much more variability between individual simulations, as can be seen from the reported standard errors. In addition, for the system with a step edge along [210], induction times were very long and in two simulations only a small volume of ice had formed after $300 \mathrm{~ns}$. Growth rates of $0.138 \AA \cdot \mathrm{ns}^{-1}$ and $0.267 \AA \cdot \mathrm{ns}^{-1}$ are observed for the surface with a terrace, or a pit, respectively.

Table 2: Average ice growth rates on different AgI (0001) surfaces with defects at $T=263 \mathrm{~K}$. Growth rates are calculated from the change of ice volume or number of ice layers as a function of time $\left(1 \AA \cdot \mathrm{ns}^{-1}=0.1 \mathrm{~m} \cdot \mathrm{s}^{-1}\right)$.

\begin{tabular}{ccc}
\hline System & Growth rate by volume $\left(\AA \cdot \mathrm{ns}^{-1}\right)$ & Growth rate by layers $\left(\AA \cdot \mathrm{ns}^{-1}\right)$ \\
\hline Perfect surface & $0.300 \pm 0.023$ & $0.859 \pm 0.062$ \\
Single vacancy & $0.306 \pm 0.017$ & $0.877 \pm 0.048$ \\
Double vacancy & $0.305 \pm 0.031$ & $0.823 \pm 0.068$ \\
Step edge [100] & $0.032 \pm 0.002$ & $0.343 \pm 0.047$ \\
Step edge [210] & $0.033 \pm 0.012$ & $0.275 \pm 0.054$ \\
Terrace & $0.138 \pm 0.032$ & $0.387 \pm 0.048$ \\
Pit & $0.267 \pm 0.016$ & $0.589 \pm 0.082$ \\
\hline
\end{tabular}

\section{Growth by ice layer thickness}

Since ice eventually grows layer-by-layer on a flat surface in periodic boundary conditions, the growth rate can also be determined by counting the number of layers of ice formed per time. To determine the number of ice layers in a given frame of the trajectory, we calculate the number density profiles along the $\mathrm{z}$ axis perpendicular to the surface for cubic and hexagonal ice, as well as interfacial and liquid water. Figure 8 shows an example of the number density of water molecules along the $\mathrm{z}$ direction during ice growth on a perfect AgI (0001) surface. Each time the sum of the densities of hexagonal and cubic ice in a number density double peak crosses the value 0.01, a new layer of ice is counted. The total ice thickness is determined by multiplying the number of layers with the thickness of an ice bilayer. The growth rates by ice layer thickness and their standard error are reported in Table 2. It should be noted that our choice of a small number density cutoff leads to a 
different definition of the growth rate compared to the volume growth rate, i.e. the signal follows more accurately the actual height of ice layers, even for hemispherical clusters, and leads to clearly higher growth rate values than the volume growth rates whenever ice does not grow layer-by-layer. Nevertheless, the relative growth rates between different systems agree reasonably well with the growth rates determined by using the volume of ice as the growth signal. For the perfect, single vacancy and double vacancy systems, the growth rates are higher than for step edge, terrace and pit systems.

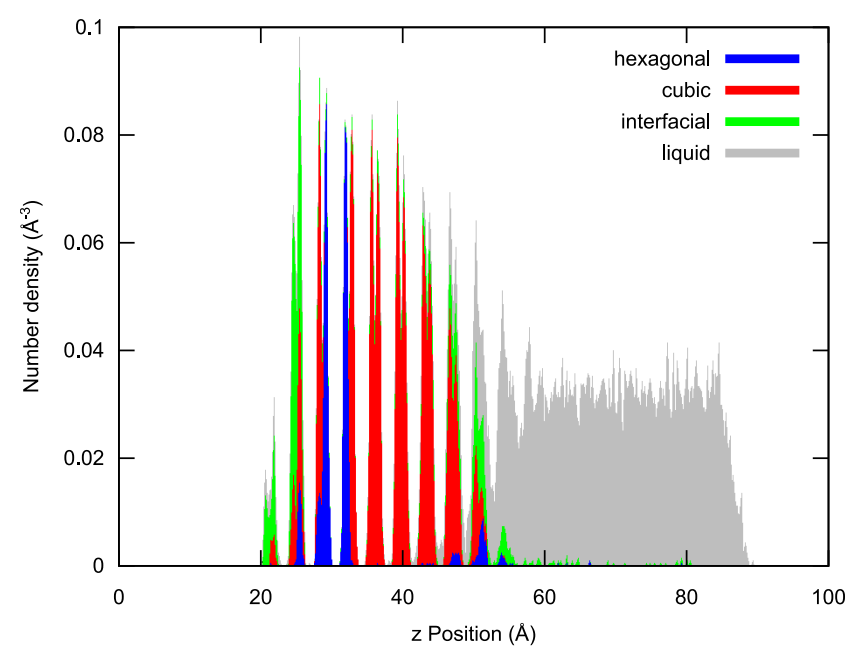

Figure 8: Number density of $\mathrm{H}_{2} \mathrm{O}$ molecules along the $\mathrm{z}$ axis perpendicular to the surface, with the respective fraction of cubic and hexagonal ice, and interfacial and liquid water, colored in red, blue, green and gray.

\section{Growth mechanisms}

During the growth of ice, stacking faults between domains of ice $I_{h}$ and $I_{c}$ will appear in a stochastic fashion. However, since we have simulated 10 individual trajectories for ice nucleation and growth on each system, we have gathered some statistics that allow us to relate the presence of a certain defect type to changes in the growth mechanism. In Figure 9, we relate changes in the growth rates to the atomistic details of the ice structure determined from the analysis with the CHILL+ algorithm, for a perfect surface, surfaces with step edges along [100] and [210], as well as a surface with a pit. Snapshots of the final configuration of 
each simulation, analysed with the CHILL+ algorithm, are also shown in Figure S1 in the Supporting Information.

On the perfect surface, once a complete bilayer of ice has formed, ice growth proceeds mostly layer-by-layer, with both ice $\mathrm{I}_{\mathrm{c}}$ and $\mathrm{I}_{\mathrm{h}}$ appearing in a stacking disordered fashion, similar to growth of pure ice. ${ }^{38}$ The growth of a single phase of ice proceeds at almost constant rate of $0.4 \AA \cdot \mathrm{ns}^{-1}$, and we will refer to this as "ideal growth" in the following. Once a stacking fault nucleates, the growth slows down until a clear grain boundary between the two phases across the periodic boundaries of the system has been established, after which the growth accelerates again. This mechanism, where the system grows alternating between two growth rates is illustrated in Figure 9a. The surface with vacancies essentially shows the same behaviour as the perfect surface. On the surface with a double vacancy we also sometimes observed the nucleation of a stacking fault at the defect site. On the surfaces with step edges, ice nuclei can appear almost simultaneously on both terraces, or separated in time, and defining induction times and growth rates is somewhat ambiguous. In every simulation, ice nucleated on the terraces away from the step edge. In principle, the first hydration layer on the upper terrace is at a similar height as the second hydration layer on the lower terrace (see Fig. 6a), and a continuous layer of ice connecting through the periodic boundaries could form at this height. However, in case the growth starts at similar times, but with different stacking of ice phases, a stacking fault above the step edge will result, hindering the transition into ideal growth, even after the initially separate volumes of ice have reached the same height (see Fig. 9a). If ice nucleates on one terrace only, the growth of ice is limited once the step edges are reached, leading to the formation of a half-cylindrical volume of ice connecting through the periodic boundaries, as shown in Figure 9c for a step edge along [100]. In this particular case, the nucleation on the lower terrace happens much later, but eventually the new volume of ice connects to the half-cylinder on the upper terrace through a stacking fault. For the systems with a pit, the nucleation happens typically outside the pit, and several layers of ice can form before ice nucleates inside the pit and eventually 
connects to the remaining volume, as shown in Figure 9d.

(a) Perfect surface
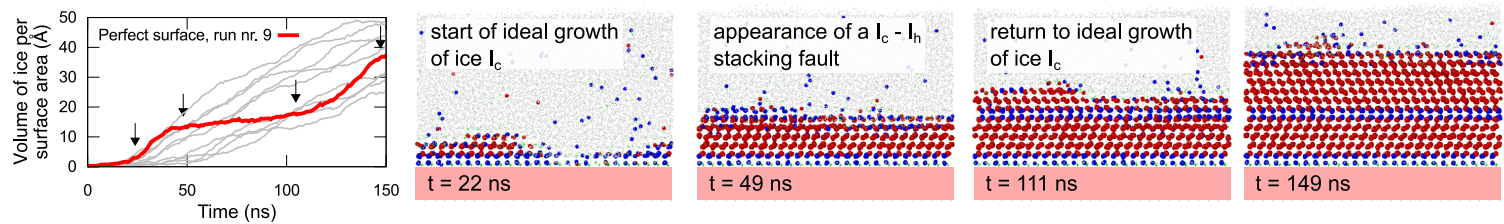

(b) Step edge along [210]
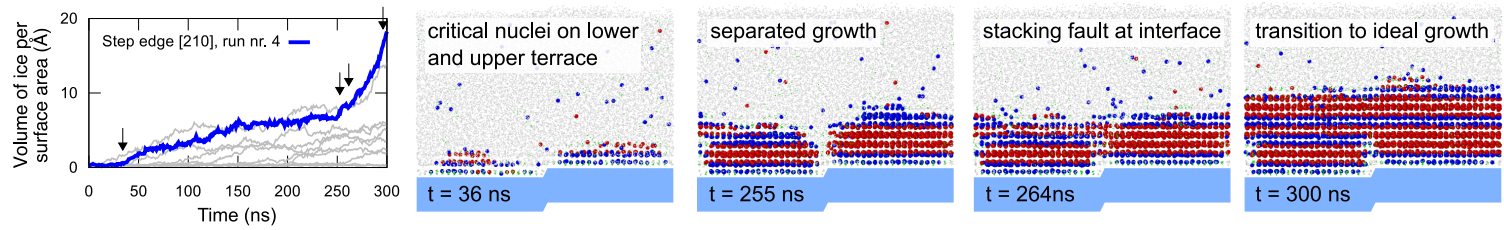

(c) Step edge along [100]
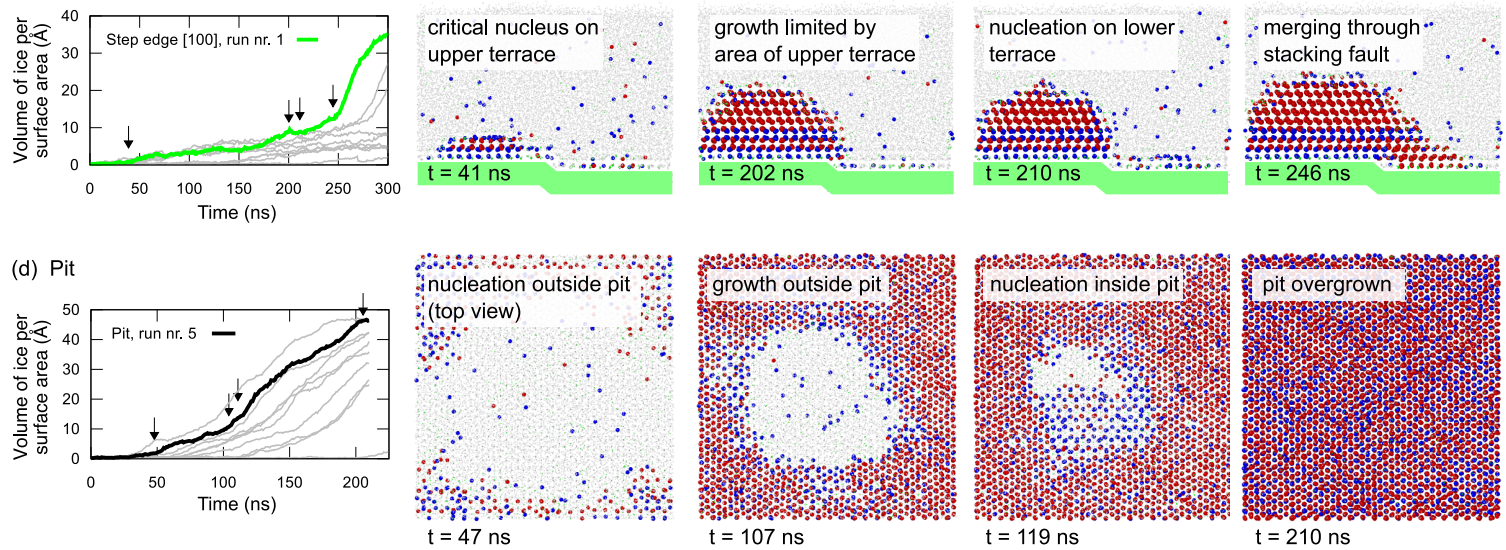

Figure 9: Examples of the atomistic details of ice growth on perfect AgI (0001) surfaces (a) and surfaces with a step edges along [210] (b) or along [100] (c), and a surface with a pit (d). The figures on the left-hand side show the ice growth curves for the 10 different simulations for each system (gray lines). One simulation run is highlighted in color, and four snapshots of this particular MD trajectory taken at times indicated by the black arrows are shown next to each figure. In these snapshots, water molecules have been color-coded as cubic ice (red), hexagonal ice (blue), interfacial (green) and liquid (gray), to illustrate the different growth mechanism. In panels (a-c) the system is shown in a side view, whereas the pit system in the bottom panel $(\mathrm{d})$ is shown in top view.

\section{Conclusions}

In this work, we studied the effect of surface defects on the heterogeneous nucleation of ice at the (0001) surface of $\beta$-AgI using atomistic molecular dynamics simulations at $T=263 \mathrm{~K}$. The presence of defects reduces both the nucleation rate and the growth rate, with a clear 
correlation between the dimensionality of the defects and the severity of the reduction: while single and double vacancies have no significant effect, the presence of step edges, terraces and pits reduces nucleation and growth rates by up to an order of magnitude. However, it is important to note that even surfaces with these last three defect types still exhibit good ice nucleation abilities at very moderate undercooling.

The simulation results show that the $\beta$-AgI (0001) surface enhances the formation of a critical nucleus by enforcing its hexagonal structure to the first hydration layer. On the perfect surface, water molecule oxygen atoms in the first hydration layer are hexagonally arranged around the protruding silver ion in the surface. In the proximity of defects such as a step edge, there are significant deviations from these ideal positions, hindering the formation of critical ice nuclei. A very simple model, where the nucleation rate on the perfect surface is scaled by the fraction of surface area available for nucleation, excluding the area around the defects, gives good agreement with the nucleation rates obtained from molecular dynamics simulations of the different surfaces with defects. However, the model obviously cannot predict differences caused by atomistic details of a defect, such as the different nucleation rates observed in molecular dynamics simulations of surfaces with step edges along [100] or [210] directions. An analysis of the interfacial hydrogen bond network reveals differences in occurrences of hexagonal (HC) and double-diamond cages (DDC) for extended defects of similar spatial extent, but fails to provide a direct explanation for the reduction in nucleation rates. However, the extended defects clearly promote the combination of $\mathrm{HC}$ and DDC at the interface, contrary to the perfect surface and surfaces with point defects, where only a single type of network is observed.

Our simulations show that the growth of ice essentially proceeds layer-by-layer once the nucleus has covered the entire interface. The rate is highest when ice grows uniformly, either as ice $I_{h}$ or $I_{c}$. The appearance of stacking faults, which reduces the growth rate, is enhanced by the presence of defects in the surface.

Finally, we would like to note that most computational studies of heterogeneous ice 
nucleation on AgI - including the present work - have considered the (0001) surface, because of its ability to nucleate ice on a time scale accessible to atomistic simulation. The fact that this surface is intrinsically unstable because of its dipole requires some brute force approaches (constraining atoms to bulk lattice positions, using a mirror image geometry to cancel the dipole field) which clearly indicates that this type of model is not a good representation of the actual AgI surfaces that are responsible for the material's remarkable ice nucleating abilities. Future studies should address this issue and put an emphasis on developing more realistic models of (0001) surfaces without a dipole, for example by considering the presence of ions in the water, ${ }^{14}$ or more complex crystal geometries ${ }^{39}$ and surface reconstructions. ${ }^{40}$

\section{Acknowledgement}

This work was supported by the National Center of Meteorology (NCM), Abu Dhabi, UAE, under the UAE Research Program for Rain Enhancement Science, the Academy of Finland Center of Excellence programme (grant no. 307331) and ARKTIKO project 285067 ICINA, the University of Helsinki, Faculty of Science ATMATH project, and ERC Grant 692891DAMOCLES. Supercomputing resources were provided by CSC-IT Center for Science, Ltd, Finland. Any opinions, findings and conclusions or recommendations expressed in this material are those of the authors and do not necessarily reflect the views of the National Center of Meteorology, Abu Dhabi, UAE, funder of the research. 


\section{Supporting Information Available}

The following files are available free of charge.

- AgI_ice_defects_SI.pdf: Ice structures analysed with the CHILL+ algorithm in the last frame of each of the 10 molecular dynamics trajectories obtained for each of the seven AgI (0001) surfaces with defects, and the top view of hydrogen bond network within the first two double layers of interfacial ice for each surface, showing hexagonal cages (HC), double-diamond cages (DDC), and mixed cages in the ice structure.

\section{References}

(1) Fitzner, M.; Sosso, G. C.; Cox, S. J.; Michaelides, A. The Many Faces of Heterogeneous Ice Nucleation: Interplay Between Surface Morphology and Hydrophobicity. J. Am. Chem. Soc. 2015, 137, 13658-13669.

(2) Kiselev, A.; Bachmann, F.; Pedevilla, P.; Cox, S. J.; Michaelides, A.; Gerthsen, D.; Leisner, T. Active Sites in Heterogeneous Ice Nucleation - the Example of K-rich Feldspars. Science 2017, 355, 367-371.

(3) Murray, B. J. Cracking the Problem of Ice Nucleation. Science 2017, 355, 346-347.

(4) Holden, M. A.; Whale, T. F.; Tarn, M. D.; O’Sullivan, D.; Walshaw, R. D.; Murray, B. J.; Meldrum, F. C.; Christenson, H. K. High-speed Imaging of Ice Nucleation in Water Proves the Existence of Active Sites. Sci. Adv. 2019, 5, eaav4316.

(5) Vonnegut, B. The Nucleation of Ice Formation by Silver Iodide. J. Appl. Phys. 1947, $18,593-595$.

(6) Marcolli, C.; Nagare, B.; Welti, A.; Lohmann, U. Ice Nucleation Efficiency of AgI: Review and New Insights. Atmos. Chem. Phys. 2016, 16, 8915-8937. 
(7) Fraux, G.; Doye, J. P. K. Note: Heterogeneous Ice Nucleation on Silver-iodide-like Surfaces. J. Chem. Phys. 2014, 141, 2.

(8) Zielke, S. A.; Bertram, A. K.; Patey, G. N. A Molecular Mechanism of Ice Nucleation on Model AgI Surfaces. J. Phys. Chem. B 2014, 119, 9049-9055.

(9) Zielke, S. A.; Bertram, A. K.; Patey, G. N. Simulations of Ice Nucleation by Model AgI Disks and Plates. J. Phys. Chem. B 2016, 120, 2291-2299.

(10) Glatz, B.; Sarupria, S. The Surface Charge Distribution Affects the Ice Nucleating Efficiency of Silver Iodide. J. Chem. Phys. 2016, 145, 211924.

(11) Prerna,; Goswami, R.; Metya, A. K.; Shevkunov, S. V.; Singh, J. K. Study of ice nucleation on silver iodide surface with defects. Mol. Phys. 2019, DOI:10.1080/00268976.2019.1657599.

(12) Shevkunov, S. V. Structure of Water Adsorbed in Slit-shaped Pores of Silver Iodide Crystal. Comput. Theor. Chem. 2016, 1084, 1-16.

(13) Tasker, P. W. The Stability of Ionic Crystal Surfaces. J. Phys. C: Solid State Phys. 1979, 12, 4977-4984.

(14) Sayer, T.; Cox, S. J. Stabilization of AgI's Polar Surfaces by the Aqueous Environment, and its Implications for Ice Formation. Phys. Chem. Chem. Phys. 2019, 21, 1454614555 .

(15) Kuang, Q.; Zheng, X.; Yang, S. AgI Microplate Monocrystals with Polar 0001 Facets: Spontaneous Photocarrier Separation and Enhanced Photocatalytic Activity. Chem. Euro. J. 2014, 20, 2637-2645.

(16) Pedevilla, P.; Fitzner, M.; Sosso, G. C.; Michaelides, A. Heterogeneous Seeded Molecular Dynamics as a Tool to Probe the Ice Nucleating Ability of Crystalline Surfaces. J. Chem. Phys. 2018, 149, 072327. 
(17) Sosso, G. C.; Li, T.; Donadio, D.; Tribello, G. A.; Michaelides, A. Microscopic Mechanism and Kinetics of Ice Formation at Complex Interfaces: Zooming in on Kaolinite. J. Phys. Chem. Lett. 2016, 7, 2350-2355.

(18) Lupi, L.; Peters, B.; Molinero, V. Pre-ordering of Interfacial Water in the Pathway of Heterogeneous Ice Nucleation Does not Lead to a Two-step Crystallization Mechanism. J. Chem. Phys. 2016, 145, 211910.

(19) Rains, C. A.; Ray, R. J.; Vashishta, P. Phase Transformations and Polytypism in Silver Iodide: A Molecular-Dynamics Study. Phys. Rev. B 1991, 44, 9228-9239.

(20) Niu, H.; Jing, Y.; Sun, Y.; Aluru, N. R. Ab Initio Based Interionic Potential for Silver Iodide. Solid State Ion. 2018, 325, 102-111.

(21) Abascal, J. L. F.; Sanz, E.; Fernández, R. G.; Vega, C. A Potential Model for the Study of Ices and Amorphous Water: TIP4P/Ice. J. Chem. Phys. 2005, 122, 234511.

(22) Hale, B. N.; Kiefer, J. Studies of $\mathrm{H}_{2} \mathrm{O}$ on beta-AgI Surfaces - An Effective Pair Potential Model. J. Chem. Phys. 1980, 73, 923-933.

(23) Stillinger, F. H.; Rahman, A. Improved Simulation of Liquid Water by Molecular Dynamics. J. Chem. Phys. 1974, 60, 1545-1557.

(24) Spoel, D. V. D.; Lindahl, E.; Hess, B.; Groenhof, G.; Mark, A. E.; Berendsen, H. J. GROMACS: Fast, Flexible, and Free. J. Comput. Chem. 2005, 26, 1701-1718.

(25) Berendsen, H. J. C.; van der Spoel, D.; van Drunen, R. GROMACS: A Message-passing Parallel Molecular Dynamics Implementation. Comput. Phys. Commun. 1995, 91, 4356.

(26) Essmann, U.; Perera, L.; Berkowitz, M. L.; Darden, T.; Lee, H.; Pedersen, L. G. A Smooth Particle Mesh Ewald Method. J. Chem. Phys. 1995, 103, 8577-8593. 
(27) Miyamoto, S.; Kollman, P. A. Settle - an Analytical Version of the Shake and Rattle Algorithm for Rigid Water Models. J. Comput. Chem. 1992, 13, 952-962.

(28) Nosé, S. A Molecular Dynamics Method for Simulations in the Canonical Ensemble. Mol. Phys. 1984, 52, 255-268.

(29) Abascal, J. L. F.; Vega, C. A General Purpose Model for the Condensed Phases of Water: TIP4P/2005. J. Chem. Phys. 2005, 123, 234505.

(30) Nguyen, A. H.; Molinero, V. Identification of Clathrate Hydrates, Hexagonal Ice, Cubic Ice, and Liquid Water in Simulations: the CHILL+ Algorithm. J. Phys. Chem. B 2015, 119, 9369-9376.

(31) Cox, S. J.; Kathmann, S. M.; Slater, B.; Michaelides, A. Molecular Simulations of Heterogeneous Ice Nucleation. I. Controlling Ice Nucleation Through Surface Hydrophilicity. J. Chem. Phys. 2015, 142, 184704.

(32) De La Pierre, M.; Raiteri, P.; Gale, J. D. Structure and Dynamics of Water at Step Edges on the Calcite $\{10 \overline{1} 4\}$ Surface. Cryst. Growth Des. 2016, 16, 5907-5914.

(33) King, S. V. Ring Configurations in a Random Network Model of Vitreous Silica. Nature 1967, 213, 1112-1113.

(34) Haji-Akbari, A.; Debenedetti, P. G. Direct Calculation of Ice Homogeneous Nucleation Rate for a Molecular Model of Water. Proc. Natl. Acad. Sci. USA 2015, 112, 1058210588.

(35) Rozmanov, D.; Kusalik, P. G. Note: Temperature Dependence of Crystal Growth of Hexagonal Ice (Ih). Phys. Chem. Chem. Phys. 2011, 141, 15501-15511.

(36) Xu, Y.; Petrik, N. G.; Smith, R. S.; Kay, B. D.; Kimmel, G. A. Growth Rate of Crystalline Ice and the Diffusivity of Supercooled Water from 126 to $262 \mathrm{~K}$. Proc. Nat. Acad. Sci. USA 2016, 113, 14921-14925. 
(37) Amaya, A. J.; Pathak, H.; Modak, V. P.; Laksmono, H.; Loh, N. D.; Sellberg, J. A.; Sierra, R. G.; McQueen, T. A.; Hayes, M. J.; Williams, G. J. et al. How Cubic Can Ice Be? J. Phys. Chem. Lett. 2017, 8, 3216-3222.

(38) Lupi, L.; Hudait, A.; Peters, B.; Grünwald, M.; Mullen, R. G.; Nguyen, A. H.; Molinero, V. Role of Stacking Disorder in Ice Nucleation. Nature 2017, 551, 218-222.

(39) Li, H.; Geelhaar, L.; Riechert, H.; Draxl, C. Computing Equilibrium Shapes of Wurtzite Crystals: The Example of GaN. Phys. Rev. Lett. 2015, 115, 449.

(40) Mora-Fonz, D.; Lazauskas, T.; Farrow, M. R.; Catlow, C. R. A.; Woodley, S. M.; Sokol, A. A. Why Are Polar Surfaces of ZnO Stable? Chem. Mater. 2017, 29, 53065320. 


\section{Graphical TOC Entry}

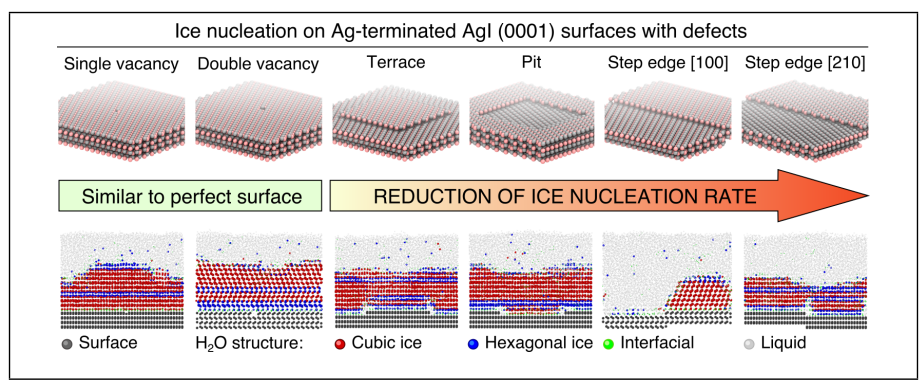

